\title{
Are isolated planetary-mass objects really isolated?
}

\section{A brown dwarf-exoplanet system candidate in the $\sigma$ Orionis cluster}

\author{
J. A. Caballero ${ }^{1,2, \star}$, E. L. Martín ${ }^{1,3}$, P. D. Dobbie ${ }^{4}$, and D. Barrado y Navascués ${ }^{5}$ \\ 1 Instituto de Astrofísica de Canarias, 38205 La Laguna, Tenerife, Spain \\ e-mail: zvezda@iac.es \\ 2 Max-Planck-Institut für Astronomie, Königstuhl 17, 69117 Heidelberg, Germany \\ 3 University of Central Florida, Department of Physics, PO Box 162385, Orlando, FL 32816-2385, USA \\ 4 Department of Physics and Astronomy, University of Leicester, University Road, Leicester LE1 7RH, UK \\ 5 LAEFF-INTA, PO Box 50727, 28080, Madrid, Spain
}

Received 2 August 2006 / Accepted 30 August 2006

\begin{abstract}
Context. Free-floating planetary-mass objects have masses below the deuterium burning mass limit at about 13 Jupiter masses, and have mostly been found in very young open clusters. Their origin and relationship to stars and brown dwarfs are still a mystery.

Aims. The recent detection by direct imaging of three giant planets at wide separation (50-250 AU) from their primaries has raised the question about the true "isolation" of planetary-mass objects in clusters. Our goal was to test the possibility that some free-floating planetary-mass objects could in fact be part of wide planetary systems.

Methods. We searched in the literature for stellar and brown-dwarf candidates members of the $\sigma$ Orionis cluster $(\sim 3 \mathrm{Ma}, \sim 360 \mathrm{pc})$ at small angular separations from published candidate planetary-mass objects. We found one candidate planetary system composed of an X-ray source, SE 70, and a planetary-mass object, S Ori 68, separated by only 4.6 arcsec. In order to assess the cluster membership of the X-ray source, we obtained mid-resolution optical spectroscopy using ISIS on the William Herschel Telescope. We also compiled additional data on the target from available astronomical catalogues.

Results. We have found that SE 70 follows the spectrophotometric sequence of the cluster and displays spectroscopic features of youth, such as lithium in absorption and chromospheric $\mathrm{H} \alpha$ emission. The radial velocity is consistent with cluster membership. Hence, SE 70 is very probably a member of the $\sigma$ Orionis cluster. The projected physical separation between SE 70 and S Ori 68 is $1700 \pm 300 \mathrm{AU}$ at the distance of the cluster. If a common proper motion is confirmed in the near future, the system would be composed of an M5-6 brown dwarf with an estimated mass of $\sim 45 M_{\text {Jup }}$ and an L5 \pm 2 giant planet with an estimated mass of $\sim 5 M_{\text {Jup }}$. It would be the widest and one of the lowest-mass planetary systems known so far.
\end{abstract}

Key words. stars: low-mass, brown dwarfs - planetary systems - open clusters and associations: individual: $\sigma$ Orionis

\section{Introduction}

Planetary-mass objects (PMOs) were directly detected for the first time in the $\sigma$ Orionis cluster and in other very young starforming regions (Zapatero Osorio et al. 2000; Lucas \& Roche 2000; Najita et al. 2000). Recent discoveries suggest that PMOs are abundant in clusters and include the detection of objects with masses potentially as low as $\sim 3$ Jupiter masses $\left(M_{\mathrm{Jup}}\right)$ (Zapatero Osorio et al. 2002c; Lucas et al. 2005). Furthermore, observations suggest that many PMOs are surrounded by discs (Luhman et al. 2005). Although cluster PMOs have masses below the deuterium-burning mass limit $\left(\sim 13 M_{\text {Jup }}\right.$; Chabrier \& Baraffe 2000), they have not achieved the "grade" of exoplanet because they appear to float freely in the intracluster medium and not be gravitationally bound to other star cluster members. However, the direct detection of planetary-mass candidates around a brown dwarf of the TW Hydrae association (Chauvin et al. 2004 - at a projected physical separation of $55 \mathrm{AU}$ ) and around the stars AB Pictoris and GQ Lupi (Chauvin et al. 2005 - 250 AU; Neuhäuser et al. 2005 - 100 AU) presents the opportunity to photometrically and spectroscopically characterize

* Alexander von Humboldt Fellow at the Max-Planck-Institut für Astronomie. exoplanets at orbital separations much larger than those indirectly detected with radial velocity surveys (the radial velocity exoplanet candidate with the largest semi-major axis is $55 \mathrm{Cnc} \mathrm{d}$, with $a=5.3 \pm 0.9 \mathrm{AU}$; Marcy et al. 2002). The discussion on how PMOs in wide orbits can be formed has been fueled by the recent discovery of the system Oph 162225-240515 $\mathrm{AB}(240 \mathrm{AU})$ in the $1 \mathrm{Ma}$-old Ophiuchus region, which may be a very low-mass brown dwarf and a PMO pair (Jayawardhana \& Ivanov 2006) or a low-mass brown-dwarf binary (Close et al. submitted).

Here, we present the first results of a survey for wide planetary systems in the $\sigma$ Orionis cluster. We have searched for candidate low-mass stellar and brown-dwarf members in close proximity to known PMOs. Our knowledge of the stellar and brown-dwarf populations of the cluster has dramatically increased in the last few years (Béjar et al. 1999, 2001, 2004 - mostly focused on very low mass stars and brown dwarfs; Scholz \& Eislöffel 2004; Caballero et al. 2004; Sherry et al. 2004; Kenyon et al. 2005; Burningham et al. 2005; Hernández et al. 2005). Prior to these studies, only massive and/or notably active stars were known in the cluster (e.g. Haro \& Moreno 1953; Warren \& Hesser 1978; Wiramihardja et al. 1989; Brown et al. 1994; Wolk et al. 1996). This cluster has become a 
favourite hunting ground for brown dwarfs and PMOs because it is nearby $\left(360_{-60}^{+70} \mathrm{pc}\right)$, young $\left(3_{-2}^{+5} \mathrm{Ma}\right)$ and free of extinction (e.g. Caballero 2005; Jeffries et al. 2006).

\subsection{SE 70 and SOri 68}

The search for candidate cluster members at projected separations less than 15 arcsec (about 5000 AU at the cluster distance) from isolated PMO candidates of $\sigma$ Orionis had been unfruitful until the recent spatial and spectral analysis of a full XMM-Newton/EPIC field in the cluster performed by Franciosini, Pallavicini \& Sanz-Forcada (2006). In their work, out of the $175 \mathrm{X}$-ray sources detected, 88 were identified with cluster member candidates extracted from the literature. In two cases, the identification was ambiguous since there were two cluster candidate members falling inside their identification radii. One of these systems, associated with the X-ray source NX 67, was formed by an intermediate M-type brown-dwarf candidate proposed by Scholz \& Eislöffel (2004), named SE 70, and by a PMO candidate, S Ori 68. NX 67 underwent a flare during the observation, with an increase in the count rate over the quiescent level by a factor of $\sim 4$ and a total duration of about $3 \mathrm{~h}$. Although Franciosini et al. (2006) did not exclude the presence of a very weak contribution from S Ori 68 to the total X-ray flux, they concluded that the bulk of it was associated with SE 70.

The planetary-mass object candidate S Ori 68 ( $\left.\sim 5 M_{\text {Jup }}\right)$, previously classified as "isolated", is about 4.6 arcsec southeast of SE $70(\theta \approx 140 \mathrm{deg})$. At the distance of the $\sigma$ Orionis cluster, the projected physical separation is $1700 \pm 300$ AU. S Ori 68 was first identified by Zapatero Osorio et al. (2000) as a freefloating planetary candidate from an $I J$ survey and follow-up $K$-band photometry. Barrado y Navascués et al. (2001) classified it as an L5.0 \pm 2.0 -type object from an optical spectrum taken with FORS at the Very Large Telescope. Although the object follows the spectrophotometric cluster sequence, the low resolution and the insufficient signal-to-noise ratio of the data did not allow them to detect spectroscopic features of extreme youth (i.e. Li I $\lambda 6707.8$ in absorption, strong asymmetric $\mathrm{H} \alpha$ emission, anomalous broadening of atomic absorption lines due to low gravity). More data are needed to confirm its membership in the $\sigma$ Orionis cluster.

The proximity between SE 70 to S Ori 68 has provided us with the motivation to now examine in more detail the physical properties of the former. We have taken intermediate-resolution optical spectroscopy and have compiled astrometric and photometric data of SE 70 to test more rigorously the hypothesis of $\sigma$ Orionis cluster membership.

\section{Observations and data mining}

\subsection{Optical spectroscopy}

On 2006 February 4, we used the red arm of ISIS at the $4.2 \mathrm{~m}$ William Herschel Telescope (WHT) at the Observatorio del Roque de los Muchachos to obtain four mid-resolution spectra of SE 70. ISIS is a high-efficiency, double-armed, mediumresolution (8-120 $\AA \mathrm{mm}^{-1}$ ) spectrograph. The detector mounted on the red arm of ISIS is a Marconi2 with 4610 pixels along the dispersion axis. The four spectra covered the wavelength range $6200-7100 \AA$ (central $\lambda=6561 \AA$ ), which allowed us to study the $\mathrm{H} \alpha$ and $\mathrm{Li}$ I $\lambda 6707.8$ spectroscopic features, and also to estimate both the spectral type and the radial velocity of the object. Nominal dispersion with the red grating with 1200 rulings $\mathrm{mm}^{-1}$ (R1200R) was $16.6 \AA \mathrm{mm}^{-1}$, which together with the 1.0 -arcsec
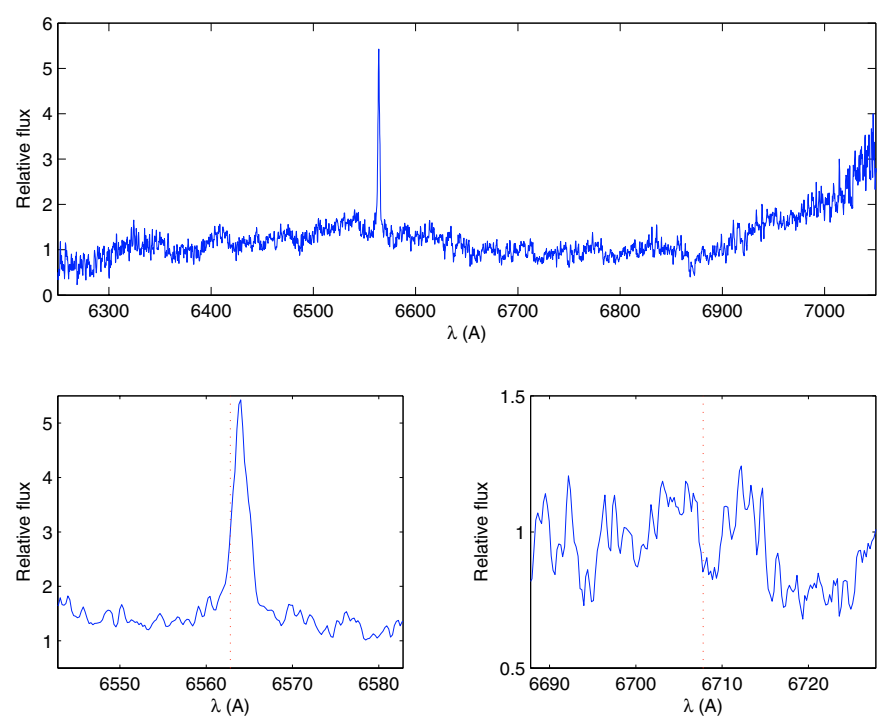

Fig. 1. Final combined spectrum of SE 70, taken with ISIS/WHT $\left(\mathrm{F}_{\lambda}\right.$, 3-pixel smoothing). Top window: whole spectral range. Bottom windows: portions of $20 \AA$ width centred in $\mathrm{H} \alpha$ (left) and Li I (right). Line identifications are marked with dotted vertical lines. The spectrum has not been corrected for heliocentric or intrinsic radial velocities.

slit led to a spectral resolution $R \sim 3000$. The seeing was good (0.6-0.8 arcsec) and the sky was fairly clear. The total exposure time of the final co-added spectrum was $4 \times 1800 \mathrm{~s}=2 \mathrm{~h}$. The data were reduced following standard procedures which included bias subtraction, flat correction, wavelength calibration (with arc spectra taken at the same position of the sky as the target) and flux normalization (we observed one flux standard star - HD 289002, B3V - just before and after SE 70). The whole wavelength interval of the final combined spectrum of SE 70 is shown in the top window of Fig. 1.

We estimated the spectral type of SE 70 at M5 1 from the calculation of the following spectral indices: PC1, I1, I2, I3 (Martín et al. 1996; Martín \& Kun 1996). All of them are based on the the ratio between the spectral fluxes at two different wavelengths, which roughly provides the slope of the spectral energy distribution in the red optical range. Calculated indices from the co-added SE 70 spectrum were compared to a grid of indices for different spectral types (early to late $\mathbf{M}$ ) and luminosity classes (V to III). The dwarf class provided the best fit. The spectral types determined for each index were in the range M4.0 to M6.5, with M5.0 being the most probable spectral type. This value is between 1.0 and 1.5 spectral subtypes earlier than expected from the $J$-band magnitude of SE 70 for a cluster member (see next section), but is consistent within the error bars. It should be noted that it is difficult to tightly constrain the spectral type from such a short wavelength interval, which allows us to use only a limited number of spectral type indicators. The strength of the titanium and vanadium oxide bands and the increasing slope of the spectrum redwards of $6900 \AA$ are further evidence of the relatively late spectral type of SE 70 .

The $\mathrm{H} \alpha \lambda 6562.8$ atomic feature is in emission. All the M-type stars and brown dwarfs in the $\sigma$ Orionis cluster with available spectroscopy display $\mathrm{H} \alpha$ in emission (see, for example, Zapatero Osorio et al. 2002a; Caballero 2006 - this fact is also applicable to all the K-type stars of the cluster). In general, this emission is due to chromospheric activity, and the spectra show pseudo-equivalent widths of the $\mathrm{H} \alpha$ line, $p E W(\mathrm{H} \alpha)$, of about $-5 \AA$. However, in a few cases, the emission is due to 
Table 1. Spectroscopic data of SE 70 and S Ori 68.

\begin{tabular}{lcc}
\hline \hline & SE 70 $^{a}$ & S Ori 68 $^{b}$ \\
\hline Sp. type & M5.0 \pm 1.0 & L5.0 \pm 2.0 \\
$p E W(\mathrm{H} \alpha)(\AA)$ & $-4.5 \pm 1.0$ & $<20$ \\
$p E W(\mathrm{Li} \mathrm{I})(\AA)$ & $+0.60 \pm 0.10$ & - \\
$V_{r}\left(\mathrm{~km} \mathrm{~s}^{-1}\right)$ & $+40 \pm 10$ & - \\
\hline
\end{tabular}

${ }^{a}$ SE 70 (IAU name: [SE2004] 70; also: NX 67, 2MASS J053838.88022801.6, DENIS J053838.9-02280, USNO-B1.0 0875-0103266).

$b$ S Ori 68 (IAU names: [BZR99] S Ori 68, [BMZ2001] S Ori J053839.1-022805). Spectral type and $\mathrm{H} \alpha$ pseudoequivalent width upper limit from Barrado y Navascués et al. (2001).

accretion from a disc, with $p E W(\mathrm{H} \alpha)$ of a few tens to a few hundred $\AA$ (Zapatero Osorio et al. 2002a,b; Caballero et al. 2006). We have measured the $p E W(\mathrm{H} \alpha)$ in the final combined spectrum of SE 70 at $-4.5 \pm 1.0 \AA$. According to the criterion defined by Barrado y Navascués \& Martín (2003), SE 70 is not an accretor. The $p E W(\mathrm{H} \alpha) \mathrm{s}$ in each of the four individual spectra range between 3.6 and $5.4 \AA$, which are consistent with the stability of the emission at the $1 \sigma$ level. The profile of the $\mathrm{H} \alpha$ emission is shown in the bottom left window of Fig. 1. The signal-tonoise ratio of the final spectrum does not allow us to detect any asymmetry.

We have also detected Li I $\lambda 6707.8$ in absorption, with a pseudo-equivalent width, $p E W(\mathrm{Li}$ I), of $+0.60 \pm 0.10 \AA$. This value is similar to those of other members of the $\sigma$ Orionis cluster with intermediate $\mathrm{M}$ spectral type (Zapatero Osorio et al. 2002a; Kenyon et al. 2005). Since objects of this spectral type destroy all their lithium through nuclear reactions in only a few megayears, it is derived that SE 70 is an extremely young M-type object with an age between 1 and $\sim 10 \mathrm{Ma}$ (the minimum and maximum ages for the $\sigma$ Orionis cluster are 1 and $8 \mathrm{Ma}$, respectively).

Using the atomic features that are visible in the wavelength interval studied, $\mathrm{H} \alpha$, Li I and some Fe I lines, and the heads of some titanium oxide bands, we have estimated the radial velocity of SE 70 at about $40 \mathrm{~km} \mathrm{~s}^{-1}$ (the heliocentric velocity correction for the mean time of observation was $-25.3 \mathrm{~km} \mathrm{~s}^{-1}$ ). Assuming a conservative uncertainty of $25 \%$, the estimated value is consistent with other more precise determinations of the mean radial velocity of the cluster found in the literature (at about 30-35 $\mathrm{km} \mathrm{s}^{-1}$ - Walter et al. 1998; Zapatero Osorio et al. 2002a; Muzerolle et al. 2003; Kenyon et al. 2005; Burningham et al. 2005; Caballero 2006; Jeffries et al. 2006 -group 1). However, if the $\mathrm{H} \alpha$ emission is not chromospheric in origin, it could be blue-/redshifted, which would introduce an additional source of uncertainty into our measurement. We have also determined the radial velocity of SE 70 with respect to several emission lines that may be adscribed to the nebulosity in the line of sight, and that are probably associated with the star-forming region. The difference between both radial velocities is null with an accuracy of $5 \mathrm{~km} \mathrm{~s}^{-1}$.

In Table 1 we summarize the measurements mentioned above (spectral type, $\mathrm{H} \alpha$ and $\mathrm{Li}$ I pseudo-equivalent widths and radial velocity of SE 70). For completeness, the spectral type and $\mathrm{H} \alpha$ pseudo-equivalent width upper limit from Barrado $\mathrm{y}$ Navascués et al. (2001) of S Ori 68 are also provided. Spectra with higher signal-to-noise ratio for both objects would be desirable for confirming membership to the $\sigma$ Orionis cluster.

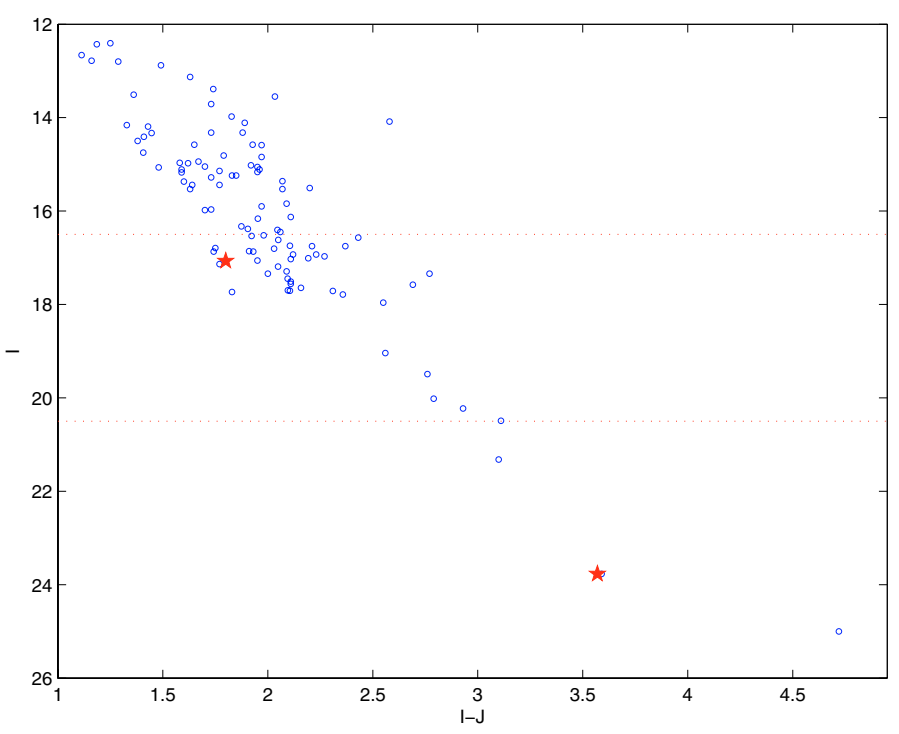

Fig. 2. $I$ vs. $I-J$ colour-magnitude diagram showing the $\sigma$ Orionis members fainter than $I=12$ mag with spectroscopic features of youth compiled by Caballero (2005), in small open circles, and SE 70 and S Ori 68, in large filled stars. Rough hydrogen (top) and deuterium (bottom) burning mass limits are shown with dotted lines.

\subsection{Additional data}

The coordinates of the optical and near-infrared counterparts of SE 70 in the 2MASS, DENIS and USNO-B1.0 catalogues match with a precision better than 0.1 arcsec. Coordinates of this target in the discovery paper by Scholz \& Eislöffel (2004) do not deviate from those of $2 \mathrm{MASS}$ by more than 0.5 arcsec. Those authors did not report SE 70 to be a photometric variable in the $I$ band and estimated an upper limit of $0.035 \mathrm{mag}$ for its photometric stability. They announced an optical magnitude of $I=16.60 \mathrm{mag}$, which led to a comparatively blue $I-J_{2 \mathrm{MASS}}$ colour for its magnitude if it is a cluster member. However, based on $I$-band data from DENIS catalogue, we determine an $I-J$ colour of $1.80 \pm 0.13 \mathrm{mag}$, which locates this source on the the bluest part of the spectrophotometric cluster sequence in the $I$ vs. $I-J$ colour-magnitude diagram, in the region of other M5-7 cluster members with lithium in absorption (Fig. 2). SE 70 was not selected as a photometric candidate during other optical searches in the area (Béjar et al. 1999, 2001; Kenyon et al. 2005). Some bright brown-dwarf cluster members with proven membership, such as the classical T Tauri substellar analogue S Ori J053825.4-024241, display quite blue colours in the red optical and had also escaped previous searches (Caballero et al. 2006).

Additional photographic $B$ - and $R$-band and [3.6]-, [4.5]-, [5.8]- and [8.0]-band photometry has been taken from the USNO-B1.0 catalogue and the IRAC/Spitzer Telescope archive, respectively (see Caballero et al., in prep., for the latter). In Table 2 we provide all the collected photometry of SE 70. The extremely red optical-infrared colours of the target support the late-M spectral type derived spectroscopically. Furthermore, the spectral energy distribution, shown in Fig. 3, is identical to those of other non-accreting M5-7 very low mass star and brown dwarf members of the $\sigma$ Orionis cluster. Figure 3 also shows the spectral energy distribution of a slightly warmer object close to the star-brown dwarf boundary (M5.0 \pm 0.5$)$ and a cooler brown dwarf (M8.5 \pm 0.5 ). Both objects display spectral features of extreme youth and are bona fide cluster members (Zapatero Osorio et al. 2002, 2003; Kenyon et al. 2005). There is no evidence of 
Table 2. Astrometric and photometric data of SE 70 and S Ori 68.

\begin{tabular}{lcc}
\hline \hline & SE 70 & S Ori 68 $^{b}$ \\
\hline$\alpha$ & 053838.89 & 053839.1 \\
$\delta$ & -022801.6 & -022805 \\
$\mu_{\alpha} \cos \delta\left(\mathrm{mas} \mathrm{a}^{-1}\right)$ & $-14 \pm 7$ & - \\
$\mu_{\delta}\left(\mathrm{mas} \mathrm{a}^{-1}\right)$ & $+8 \pm 3$ & - \\
\hline$B$ & $20.68 \pm 0.56$ & - \\
$R$ & $19.05 \pm 0.24$ & - \\
$I$ & $17.07 \pm 0.12$ & $23.77 \pm 0.17$ \\
$J$ & $15.27 \pm 0.04$ & $20.2 \pm 0.3$ \\
$H$ & $14.73 \pm 0.05$ & - \\
$K_{\mathrm{s}}$ & $14.43 \pm 0.07$ & $18.4 \pm 0.3$ \\
{$[3.6]$} & $14.06 \pm 0.14$ & - \\
{$[4.5]$} & $14.07 \pm 0.17$ & - \\
{$[5.8]$} & $13.76 \pm 0.19$ & - \\
{$[8.0]$} & $13.8 \pm 0.3$ & - \\
\hline
\end{tabular}

${ }^{a}$ Coordinates $\alpha$ and $\delta$ (J2000) and $J H K_{\mathrm{s}}$ magnitudes from 2MASS (Cutri et al. 2003); $B$ (averaging two detections) and $R$ magnitudes (averaging two double detections) and tangential velocities $\mu_{\alpha}$ and $\mu_{\delta}$ from USNO-B1.0 catalogue (Monet et al. 2003); I magnitude from DENIS catalogue (The DENIS Consortium); Spitzer-band magnitudes from Caballero et al. (in prep.).

$b$ Coordinates $\alpha$ and $\delta(\mathrm{J} 2000)$ and $I J K$ magnitudes from Zapatero Osorio et al. (2000).

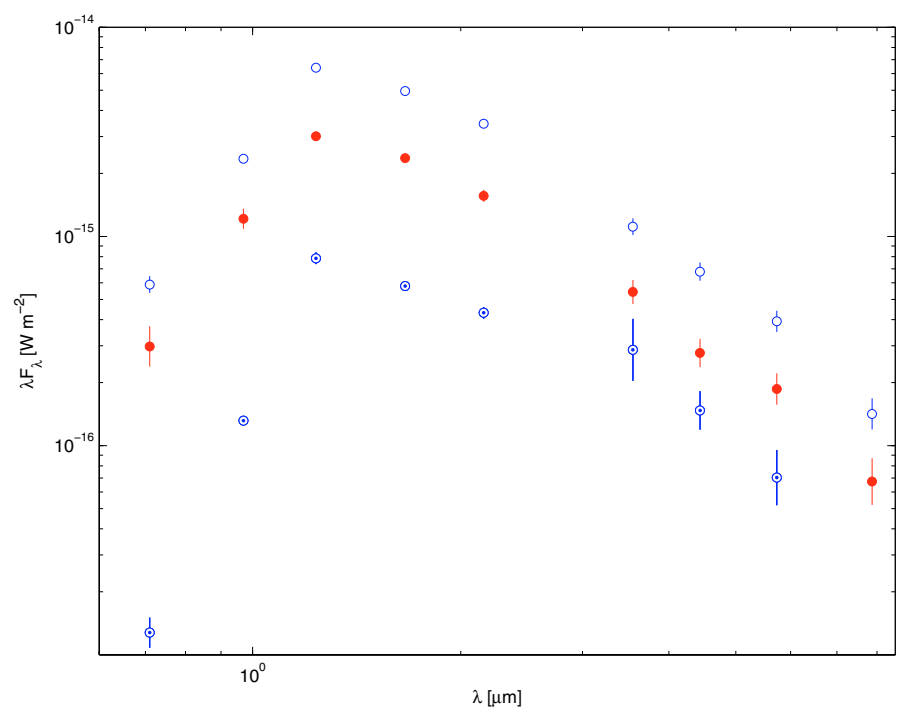

Fig. 3. Spectral energy distributions of SE 70 from the optical $R$ band to $8.0 \mu \mathrm{m}$ (filled circles, "•") The spectral energy distributions of two very low-mass cluster members with lithium in absorption and other cluster membership indices are also given for comparison. They are S Ori J053911.4-023333, M5.0 \pm 0.5 (open circles “o”), and S Ori 45, M8.5 \pm 0.5 (solar symbol, “ $\odot ”)$.

any surrounding disc at wavelengths longer than $2.0 \mu \mathrm{m}$ from the shape of the spectral energy distribution of SE 70. Redwards of $1 \mu \mathrm{m}$, it provides a reasonable fit to that of a black body at $360 \mathrm{pc}$ with $T_{\text {eff }}=3200 \mathrm{~K}, R=0.42 R_{\odot}$ (bluewards of $1 \mu \mathrm{m}$ there are strong water and metallic oxide bands that affect the continuum). The estimated effective temperature is consistent with the M5-6 spectral type determination.

Table 2 also provides the astrometry of SE 70. Coordinates indicate that it is located at about 8 arcmin to the $09.5 \mathrm{~V} \mathrm{star}$ that gives the name to the cluster, $\sigma$ Ori A (and which is roughly the centre of mass of the cluster). Tangential velocities in $\alpha$ and $\delta$ have been taken from the USNO-B1.0 catalogue. The difference between the proper motions of SE 70 and $\sigma$ Ori A is as large as $\left(\Delta \mu_{\alpha}, \Delta \mu_{\delta}\right)=(-19 \pm 7,+8 \pm 3)$ mas a $^{-1}$. These values are several times larger than the cluster velocity dispersion. The automatic proper motion determination at the faint optical magnitudes of SE 70, fainter than the completeness BR-band magnitudes of the USNO-B1.0 catalogue, may lead to unreliable tangential velocities or to underestimated error bars. This is probably the case here. A simple inspection of the tangential velocity values of several faint spectroscopically confirmed members of the $\sigma$ Orionis cluster supports this hypothesis (they also display proper motions that are abnormally different from zero).

\section{Discussion}

Except for the unreliable astrometric measurement, all the studied astrometric parameters indicate that SE 70 is a bona fide nonaccreting $\sigma$ Orionis cluster member: it displays X-ray emission, chromospheric $\mathrm{H} \alpha$ emission, $\mathrm{Li}$ I in absorption and radial velocity similar to those of the cluster and of the nebulosity, and lies on the spectrophotometric cluster sequence. The derived spectral type is on the borderline between very low-mass stars and brown dwarfs in $\sigma$ Orionis. However, the $J$-band magnitude of SE 70, which marks the peak in the spectral energy distribution, is about 1.0 mag fainter than those of the most massive brown dwarfs in the cluster (at the hydrogen-burning mass limit, $\sim 75 M_{\text {Jup }}$; Béjar et al. 2001; Caballero et al. 2004). Following our procedure for mass determination in the substellar domain, detailed in Caballero (2006) ${ }^{1}$, we estimate SE 70 to be a brown dwarf with a mass of $45 M_{\text {Jup }}$. Hence, S Ori 68 and SE 70 may form a system whose masses resemble those of the Chauvin et al. (2004) 2MASS J1207334-393254 system (about $45+5 M_{\text {Jup }}$ in the case of SE $70+S$ Ori 68 versus about $25+5 M_{\text {Jup }}$ in the case of 2M1207-39 + 2M1207-39b). However, the projected physical separation of our hypothetical $\sigma$ Orionis planetary system is about 30 times larger than that of 2M1207-39, and about seven times larger than that of AB Pic and Oph 162225-240515. Due to the low mass and the wide separation of the SE $70+$ S Ori 68 system, it has the lowest gravitational binding energy among the presumed planetary systems. Given the relatively high spatial density of cluster members in $\sigma$ Orionis and the weak bounding between SE 70 and S Ori 68, it seems dubious that the system can survive the tidal field within the cluster. The existence of a binary system with these characteristics is evidently a challenge for present ultra low-mass star forming scenarios, which predict tighter and more massive binary/planetary systems.

There is the possibility of the pair being a visual projection of two unrelated cluster members at slightly different heliocentric distances and hence not being a true binary system. The statistical analysis performed by Caballero (in prep.), who has studied the spatial distribution of all the stellar population and the highest-mass domain of the brown-dwarf population in the $\sigma$ Orionis cluster, indicates that this is unlikely. He has shown that $95 \%$ of the studied candidate cluster members at angular separations to $\sigma$ Ori A between 5 and 10 arcmin have their nearest neighbourhood at more than 10.9 arcsec (the SE $70+$ S Ori 68 system is at about 8 arcmin to the cluster centre). Among the more than 400 candidate cluster members studied from a $2 \mathrm{MASS}$ + DENIS correlation, none has a nearest

\footnotetext{
1 The mass is derived from the comparison between theoretical luminosities of Chabrier et al. (2000) and the luminosities of the objects. The latter are computed from the $J$-band absolute magnitude and an $I-J$ colour-dependent bolometric correction.
} 


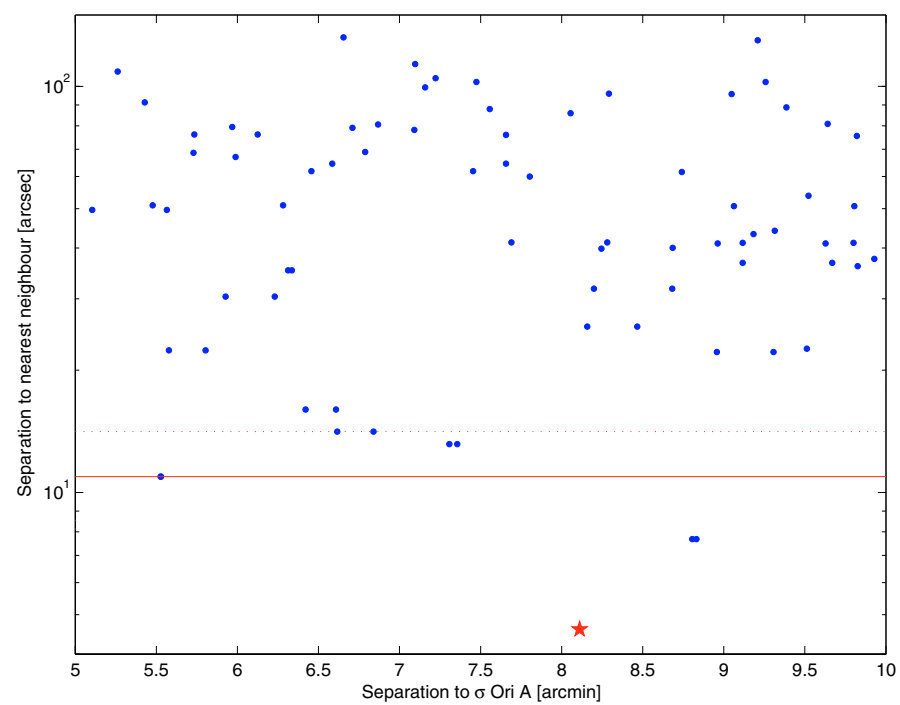

Fig. 4. Angular separation to the nearest neighbour vs. separation to the centre of the $\sigma$ Orionis cluster. Small dots represent the star and massive brown-dwarf photometric member candidates in the study by Caballero (in prep.), while the large filled stars represent the SE $70+$ S Ori 68 system. Low-mass brown-dwarf and IPMO cluster member candidates are not included. Horizontal lines denote the 90 (dotted) and 95\% (solid) confidence limits below which the probabilities for a pair at 5 to 10 arcmin to $\sigma$ Ori A to be a visual alineation are less than 10 and $5 \%$, respectively. There are only two systems below the $95 \%$ confidence limit: [W96] 4771-899 AB + C and SE $70+$ S Ori 68. The results from 0 to 30 arcmin to the cluster centre and the description of the spatial distribution study will be given in Caballero (in prep.).

neighbour closer than 7.1 arcsec, while the separation between SE 70 and S Ori 68 is only 4.6 arcsec. Figure 4 illustrates this discussion, and indicates that the SE $70+$ S Ori 68 pair has less than about $1 \%$ probability to be a chance visual alignment. There are stellar pairs in the $\sigma$ Orionis cluster with smaller separations between components, but they are spectroscopic binaries (e.g. OriNTT 429; Lee et al. 1994) or very tight binaries unresolved by 2 MASS or DENIS (e.g. $\sigma$ Ori A and $\sigma$ Ori B -0.250 arcsec; $\sigma$ Ori $\mathrm{AB}$ and $\sigma$ Ori IRS $1-3.32 \pm 0.15$ arcsec; HD $37525 \mathrm{~A}$ and HD $37525 \mathrm{~B}-0.47 \pm 0.04 \mathrm{arcsec}$; [W96] 4771-899 A and [W96] 4771-899 B - $0.40 \pm 0.08$ arcsec; Caballero 2005) ${ }^{2}$.

It is not the aim of this paper to prove beyond doubt the cluster membership of the planetary-mass candidate S Ori 68 . In order to do that, high-quality mid-resolution near-infrared spectra of S Ori 68 should be taken to detect low-gravity spectral signatures in the atomic absorption lines of alkalis, such as sodium or potassium. These signatures have already been found in the spectra of several young brown dwarfs (e.g. Gorlova et al. 2003). However, such studies require significant amounts of observing time with instruments such as LRIS at the Keck Observatory. Given the extraordinary faintness of S Ori 68 in the red optical range $(I \sim 24 \mathrm{mag}, R>26-27 \mathrm{mag}$; S Ori 68 has the same $I-$ and $J$-band magnitudes as SOri 66, an intermediate-L planetary-mass object in $\sigma$ Orionis with $\mathrm{H} \alpha$ in moderate emission), the detection of lithium in absorption in an optical spectrum is impractical with present instrumentation.

2 The tight pair [W96] 4771-899 AB and [W96] 4771-899 C (aka S Ori J053847.7-022711, at $\rho=7.63 \pm 0.10$ arcsec) is in fact a triple stellar system. There is an additional binary system candidate formed by a very low-mass star, S Ori J0539268-026614, and a faint brown dwarf at $4.4 \operatorname{arcsec}(J=16.21 \pm 0.09 \mathrm{mag}$, not found in 2MASS or DENIS catalogues). Both of them show Li I in absorption (Zapatero Osorio, et al. in prep.).
Further ultra-accurate proper motion studies are needed to verify that both S Ori 68 and SE 70 form a common proper motion system. The Ori OB1b Association lies in the direction of the solar antapex. Hence, the tangential velocities of the stellar members of the association are very low, less than 5 mas a $^{-1}$ according to de Zeeuw et al. (1999). Confirmation of the common proper motion would require an accuracy of a few hundred milliarsec. While such accurate measurements on very faint objects could be obtained with the Hubble Space Telescope if a large enough time baseline is used, realistically we will have to wait for the technology of the near future, such as the Large Binocular Telescope and the LINC-NIRVANA instrument (Herbst et al. 2004). We note that these measurements could not be obtained with the GAIA satellite, because of faintness of targets in the optical.

Radial velocity measurements of the pair obtained with current or future near-infrared high-resolution spectrographs attached to $\geq 10 \mathrm{~m}$-class telescopes, like NIRSPEC/Keck or NAHUAL/Gran Telescopio Canarias (Martín et al. 2005), might be able to constrain the binding status of the SE $70+$ S Ori 68 system. However, these measurements must be more precise than $1 \mathrm{~km} \mathrm{~s}^{-1}$ to disentangle the common proper motion of the hypothetical binary system and the velocity dispersion of the $\sigma$ Orionis cluster (that is of the order of $2.4 \mathrm{~km} \mathrm{~s}^{-1}-$ Caballero 2005). To illustrate the capabilities of NIRSPEC/Keck, Martín et al. (2006) reached a precision of $360 \mathrm{~m} \mathrm{~s}^{-1}$ in the radial velocity determination of the M9V-type brown dwarf LP 944-20.

\section{Summary}

We report on the analysis of a probable brown dwarf-exoplanet system in the $\sigma$ Orionis cluster, consisting of SE 70 and S Ori 68. The projected physical separation between them is $1700 \pm 300 \mathrm{AU}$ at the distance to the cluster. We have derived the spectral type of the primary (in the M5-6 interval) and found spectroscopic features typical in other young members of the $\sigma$ Orionis cluster: lithium in absorption, $\mathrm{H} \alpha$ in (probably chromospheric) emission and radial velocity similar to the mean radial velocity of the cluster. Franciosini et al. (2006) previously found the primary to be an X-ray emitter that underwent a flare during their observations. From an analysis of the spatial distribution of the cluster, if S Ori 68 is also a cluster member, there is about a $99 \%$ probability that they form a real planetary system. If this is the case, not all "isolated" planetary-mass objects are really isolated, and some planetary systems may have separations as wide as those of binary stellar systems.

Acknowledgements. We thank Víctor J. S. Béjar, Rafael Rebolo, María Rosa Zapatero Osorio and Jack Lucentini for helpful comments. We also thank M. F. Sterzik for suggestions that have improved the quality of the manuscript and for his fast refereeing. Partial financial support was provided by the Spanish Ministerio de Ciencia y Tecnología project AYA2005-06453 of the Plan Nacional de Astronomía y Astrofísica and by NSF grant AST0440520. Based on observations made with the William Herschel Telescope operated on the island of La Palma by the Isaac Newton Group in the Spanish Observatorio del Roque de Los Muchachos of the Instituto de Astrofísica de Canarias. This work is based in part on observations made with the Spitzer Space Telescope, which is operated by the Jet Propulsion Laboratory, California Institute of Technology under a contract with NASA.

\section{References}

Barrado y Navascués, D., Zapatero Osorio, M. R., Béjar, V. J. S., et al. A\&A, 377, L9

Barrado y Navascués, D., \& Martín, E. L. 2003, AJ, 126, 2997

Béjar, V. J. S., Zapatero Osorio, M. R., \& Rebolo, R. 1999, ApJ, 521, 671

Béjar, V. J. S., Martín, E. L., Zapatero Osorio, M. R., et al. 2001, ApJ, 556, 830 
Béjar, V. J. S., Caballero, J. A., Rebolo, R., Zapatero Osorio, M. R., \& Barrado y Navascués, D. 2004, Ap\&SS, 292, 339

Brown, A. G. A., de Geus, E. J., \& de Zeeuw, P. T. 1994, A\&A, 289, 101

Burningham, B., Naylor, T., Littlefair, S. P., \& Jeffries, R. D. 2005, MNRAS, 356,1583

Caballero, J. A. 2005, Astron. Nachr., 326, 1007

Caballero, J. A. 2006, Ph.D. thesis, Universidad de La Laguna

Caballero, J. A., Béjar, V. J. S., Rebolo, R., \& Zapatero Osorio, M. R. 2004, A\&A, 424, 857

Caballero, J. A., Martín, E. L., Zapatero Osorio, M. R., et al. 2006, A\&A, 445, 143

Chabrier, G., \& Baraffe, I. 2000, ARA\&A, 38, 337

Chabrier, G., Baraffe, I., Allard, F., \& Hauschildt, P. 2000, ApJ, 542, 464

Chauvin, G., Lagrange, A.-M., Dumas, C., Zuckerman, et al. 2004, A\&A, 425, L29

Chauvin, G., Lagrange, A.-M., et al. 2005, A\&A, 438, L29

Close, L. M., Zuckerman, B., Song, I., et al. 2006, ApJ, submitted, [arXiv: astro-ph/0608574]

Cutri, R. M., Skrutskie, M. F., van Dyk, S., et al. 2003, VizieR On-line Data Catalog: II/246. Originally published in University of Massachusetts and Infrared Processing and Analysis Center (IPAC/California Institute of Technology)

Franciosini, E., Pallavicini, R., \& Sanz-Forcada, J. 2006, A\&A, 446, 501

Gorlova, N. I., Meyer, M. R., Rieke, G. H., \& Liebert, J. 2003, ApJ, 593, 1074

Haro, G., \& Moreno, A. 1953, BOTT, 1g, 11

Herbst, T. M., Ragazzoni, R., Eckart, A., \& Weigelt, G. 2004, SPIE, 5492, 1461

Hernández, J., Calvet, N., Hartmann, L., et al. 2005, AJ, 129, 856

Jeffries, R. D., Maxted, P. F. L., Oliveira, J. M., \& Naylor, T. 2006, MNRAS, 371, L6

Jayawardhana, R., \& Ivanov D. 2006, Science, 313, 1279

Kenyon, M. J., Jeffries, R. D., Naylor, T., Oliveira, J. M., \& Maxted, P. F. L. 2005, MNRAS, 356, 89

Lee, C. W., Martín, E. L., \& Mathieu, R. D. 1994, AJ, 108, 1445
Lucas, P. W., \& Roche, P. F. 2000, MNRAS, 314, 858

Lucas, P. W., Roche, P. F., \& Tamura, M. 2005, MNRAS, 361, 211

Luhman, K. L., D'Alessio, P., Calvet, N., et al. 2005, ApJ, 620, L51

Marcy, G. W., Butler, R. P., Fischer, D. A., et al. 2002, ApJ, 581, 1375

Martín, E. L., \& Kun, M. 1996, A\&AS, 116, 467.

Martín, E. L., Rebolo, R., \& Zapatero Osorio, M. R. 1996, ApJ, 469, 706

Martín, E. L., Guenther, E., Barrado y Navascués, D., et al. 2005, AN, 326, 1015

Martín, E. L., Guenther, E., Zapatero Osorio, M. R., Bouy, H., \& Wainscoat, R. 2006, ApJ, 644, L75

Monet, D. G., Levine, S. E., Casian, B., et al. 2003, AJ, 125, 984

Muzerolle, J., Hillenbrand, L., Calvet, N., Briceño, C., \& Hartmann, L. 2003, ApJ, 592, 266

Najita, J. R., Tiede, G. P., \& Carr, J. S. 2000, ApJ, 541, 977

Neuhäuser, R., Guenther, E. W., Wuchterl, G., et al. 2005, A\&A, 435, L13

Scholz, A., \& Eislöffel, J. 2004, A\&A, 419, 249

Sherry, W. H., Walter, F. M., \& Wolk, S. J. 2004, AJ, 128, 2316

Walter, F. M., Wolk, S. J., \& Sherry, W. 1998, ASP Conf. Ser., 154, CD-1793

Warren, W. H. Jr., \& Hesser, J. E. 1978, ApJS, 36, 497

Wiramihardja, S. D., Kogure, T., Yoshida, S., Ogura, K., \& Nakano, M. 1989, PASJ, 41, 155

Wolk, S. J. 1996, Ph.D. Thesis, State Univ. New York at Stony Brook

Zapatero Osorio, M. R., Béjar, V. J. S., Martín, E. L., et al. 2000, Science, 290, 103

Zapatero Osorio, M. R., Béjar, V. J. S., Pavlenko, Ya., et al. 2002a, A\&A, 384, 937

Zapatero Osorio, M. R., Béjar, V. J. S., Martín, E. L., Barrado y Navascués D., \& Rebolo, R. 2002b, ApJ, 569, L99

Zapatero Osorio, M. R., Béjar, V. J. S., Martín, E. L., et al. 2002c, ApJ, 578, 536

Zapatero Osorio, M. R., Caballero, J. A., Béjar, V. J. S., \& Rebolo, R. 2003, A\&A, 408, 663

de Zeeuw, P. T., Hoogerwerf, R., de Bruijne, J. H. J., Brown, A. G. A., \& Blaauw, A. $1999, \mathrm{AJ}, 117,354$ 